\title{
BoCluSt: Bootstrap Clustering Stability Algorithm for Community Detection
}

\author{
Carlos Garcia* \\ CIBUS Universidade de Santiago, Campus Sur, 15782 Santiago de Compostela, Galiza, Spain \\ * carlos.garcia.suarez@usc.es
}

\section{Abstract}

The identification of modules or communities in sets of related variables is a key step in the analysis and modeling of biological systems. Procedures for this identification are usually designed to allow fast analyses of very large datasets and may produce suboptimal results when these sets are of a small to moderate size. This article introduces BoCluSt, a new, somewhat more computationally intensive, community detection procedure that is based on combining a clustering algorithm with a measure of stability under bootstrap resampling. Both computer simulation and analyses of experimental data showed that BoCluSt can outperform current procedures in the identification of multiple modules in data sets with a moderate number of variables. In addition, the procedure provides users with a null distribution of results to evaluate the support for the existence of community structure in the data. BoCluSt takes individual measures for a set of variables as input, and may be a valuable and robust exploratory tool of network analysis, as it provides 1) an estimation of the best partition of variables into modules, 2) a measure of the support for the existence of modular structures, and 3) an overall description of the whole structure, which may reveal hierarchical modular situations, in which modules are composed of smaller sub-modules.

Published: June 3, 2016

Copyright: @ 2016 Carlos Garcia. This is an open access article distributed under the terms of the Creative Commons Attribution License, which permits unrestricted use, distribution, and reproduction in any medium, provided the original author and source are credited.

Data Availability Statement: Data are available from the NCBI database, accession GSE24729 (http:// www.ncbi.nIm.nih.gov/geo/query/acc.cgi?acc= GSE24729).

Funding: This work was funded by Ministerio de Economía and Competitividad (URL: www.mineco. gob.es/; grant number: CGL2012-39861-C02-01). The funders had no role in study design, data collection and analysis, decision to publish, or preparation of the manuscript.

Competing Interests: The authors have declared that no competing interests exist.

\section{Introduction}

Complex systems are often modeled and analyzed as networks of related elements (nodes) connected by edges or links representing the relationship between them [1, 2]. In Biology, many of these networks show a modular structure: nodes can be grouped into communities or modules with a dense web of links among them, but with thin links between nodes in different modules [3-6]. This has been observed in gene expression networks [7, 8], protein-protein interactions [9], metabolic $[10,11]$ and developmental $[12,13]$ pathways, and also in species interactions in ecosystems $[14,15]$.

The building of models for these networks may require the use of module identification (also called community detection) procedures. Many such procedures have been proposed (see [16] for an ample review). Some are confirmatory, requiring a prior knowledge of module demarcations or at least of the number of modules present (e.g., $[17,18])$. Other procedures ([19-21]) are exploratory and unsupervised, making no assumptions about modules and trying 
to identify the partitions among nodes that maximize some criterion of modular structure. Some of them consider heterogeneity in link weight, which may be fundamental for the understanding of the whole network [22]. By different means many try to maximize modularity, a measure of the density of links among nodes in the same module relative to that between nodes in different modules [23]:

$$
Q=\frac{1}{4 m} \sum_{i j}\left(A_{i j}-\frac{k_{i} k_{j}}{2 m}\right) \delta\left(c_{i}, c_{j}\right)
$$

where $A_{i j}=1$ if a link connects nodes $i$ and $j$ and $A_{i j}=0$ otherwise, $k_{i}$ is the degree of (number of links connected to) node $i, m$ is the total number of links in the network, and the function $\delta$ yields 1 when modes $i$ and $j$ are in the same module and 0 otherwise. Furthermore, other procedures, called divisive, delete network links so that existing clusters get disconnected [24]; others are based on random walks, in which a walker moving randomly along the network edges is expected to make many repeated visits to variables in the same cluster, given the high density of edges among them [25]; others are based on statistical inference, i. e., they try to measure how well theoretical models of the network community structure fit the observed data [26], etc.

Given that many community detection procedures were designed to analyze very large networks in a reasonable time, they try to minimize the amount of resources required to perform their task, i. e., to minimize computational complexity. They also tend to work better when networks are sparse, i. e., when the overall link density is low, so that the number of links is similar to that of nodes [16]. However, more computing intensive methods can be used in the case of small to moderate sized data sets. Among these there are methods using stability as the criterion to validate module partitions [27,28]. A clustering algorithm is applied to a series of data samples and the solution showing the highest replicability is taken as that corresponding to the true cluster structure. Some of the procedures based on this approach [29-31] generate the series of data samples by splitting the available data into random sub-samples, while others $[28,32]$ generate full-sized data samples by bootstraping. In contrast with most community detection procedures, which take between-node link information as a fixed input, stability based procedures use each generated sample to obtain information about these links, so that their results depend on the quality of the information on the links between nodes, or the estimation precision thereof. Of course, The robustness of module allocations may depend heavily on this quality [23]. This may not be critical when the aim of the analysis is the identification of large-scale community structures in big data sets, but could become so when the focus is on the allocation of particular variables to particular modules, as in the smaller networks considered in the analyses of gene regulation pathways and signal transduction cascades. In addition, stability procedures are robust in the sense that they use no stringent definitions of partition quality and they markedly reduce biases related to the spatial distribution of modules or the models about the data used by clustering algorithms [28]. However, these procedures can be demanding on computing resources, partly because they measure stability by making pairwise comparisons between module partitions obtained in different generated data samples [33], so the number of comparisons increases rapidly with the number of these samples.

This article introduces BoCluSt, a new stability based procedure for module identification in sets of correlated variables that is less computationally demanding than previous stability methods because it measures this stability by calculating the variance of a single vector of module coincidences instead of making multiple comparisons between generated samples. An input file containing individual observations for these variables is subjected to bootstrap resampling combined with a clustering algorithm, and the clustering solution showing the highest stability under resampling is selected. The procedure also uses resampling to generate a 
distribution of results under the null situation of no correlation between variables, which makes it possible to explicitly evaluate the support for the existence of community structure in the data. Computer simulation and the analysis of gene expression data show that, for data sets of a small to moderate size, BoCluSt tends to be superior to community detection procedures for which software is available, and that it can also detect the existence of hierarchical modular structures.

\section{Materials and Methods Implementation of BoCluSt}

For a dataset of $n$ variables $x_{i}(i=1, \ldots, n)$, measured on $m$ records, a clustering method is applied to obtain partitions into $\mathrm{p}$ clusters $(2 \leq p \leq n-1)$; in the present implementation: $\mathrm{k}$ means using the R kmeans function [34], an obvious choice, as it is the simplest and most popular clustering algorithm [35]; as an alternative using 1 -absolute Pearson correlations as distances between variables, there is also the option to use the pam function-Partition Around Medoids clustering algorithm- from the R package cluster [36]). Because of its use of the kmeans algorithm, BoCluSt imposes no limits to link density in the data. Since all pairwise links between variables are considered, it works at maximum link density.

For a $p$-cluster partition, the "coincidence matrix" denoted by $\boldsymbol{C}_{\boldsymbol{p}}=\left[\boldsymbol{C}_{\boldsymbol{p} i j}\right](i, j=1, \ldots, n)$ is defined as follows:

1. $C_{p i j}=1$ if $x_{i}$ and $x_{j}$ are in the same cluster.

2. $C_{p i j}=0$ if $x_{i}$ and $x_{j}$ are not in the same cluster.

3. by agreement $C_{p i i}=1 ; i=1, \ldots, n$.

Consequently, the matrix $\boldsymbol{C}_{\boldsymbol{p}}$ is symmetric, i. e. $\boldsymbol{C}_{\boldsymbol{p} i j}=\boldsymbol{C}_{\boldsymbol{p} j \mathrm{i}}$. The $\left(n^{2}-n\right) / 2$ relevant, nonredundant (condition iii is irrelevant) elements in $C_{p}$ are stored in vector $\boldsymbol{r}_{\boldsymbol{p}}$, and the stability of each of the $n-2$ cluster analyses is tested by bootstrap resampling of the individual observations in the original dataset to generate new full-size data sets and obtain new $n-2$ vectors ${ }^{B} \boldsymbol{r}_{\boldsymbol{p}}$, where $B$ denotes the ordinal number of bootstrap $(B=1,2,3, \ldots)$. For each bootstrap resampling $B$, the analysis of the distribution of the variables into $p$ clusters gives us a new vector ${ }^{B} r_{p}$ and then the sequence of vectors $\left\{{ }^{1} \boldsymbol{r}_{\boldsymbol{p}},{ }^{2} \boldsymbol{r}_{\boldsymbol{p}}, \ldots,{ }^{B} \boldsymbol{r}_{\boldsymbol{p}}, \ldots\right\}$.

If a real, detectable module structure existed in the data, cluster analyses considering the appropriate number of clusters-modules would tend to allocate variables in the same clusters in all resamples, so that the variance across resamples would be low for each element in ${ }^{B} r_{p}$. A given pair of variables $x_{i}, x_{j}(i \neq j)$ would tend to be either in the same cluster, the corresponding element value ${ }^{B} \boldsymbol{r}_{\boldsymbol{p} i j}$ being equal to1 in most of resamples, i. e. ${ }^{B} \boldsymbol{r}_{\boldsymbol{p}}{ }_{i j}=1$ for almost all $B$. However, if $x_{i}, x_{j}(i \neq j)$ tend to be in different clusters, then ${ }^{B} \boldsymbol{r}_{\boldsymbol{p}}{ }_{i j}=0$ in most of resamples. In analyses considering wrong numbers of clusters -or analyses of data with no community structure-, each bootstrap replicate would result in clusters containing random combinations of variables, and therefore in high variance of the elements of ${ }^{B} \boldsymbol{r}_{\boldsymbol{p}}$ across bootstraps. In BoCluSt, the variance of the vector corresponding to each $p$-partition, $\operatorname{Var}\left({ }^{B} \boldsymbol{r}_{\boldsymbol{p}}\right)$, is calculated as the sum of the variances of its elements across resamples. The partition resulting in the minimum sum of variances (i. e., the number of clusters $p$ providing the most stable ${ }^{B} \boldsymbol{r}_{\boldsymbol{p}}$ ) would constitute the best estimate of the module structure in the original dataset.

However, the distribution of this sum of variances is not independent of the number of clusters considered in the successive $n-2$ cluster analyses. To correct for this effect, the sums of variances are made relative to their expected values in a null situation with the same number of clusters and a lack of correlation between variables. This is obtained by randomizing the 
observed variable values independently across individuals, so that while univariate distributions are maintained, any correlation between variables disappears. The resulting null distribution for the sum of variances also makes it possible to evaluate the amount of evidence for modular structure in a particular data set. The corrected sum of variances ("variance criterion" in what follows) is used as an optimal partition criterion.

A summary of the steps taken is:

1. A series of bootstrap samples is taken from the original dataset of $m$ records of $n$ variables.

2. The clustering algorithm is applied to each sample to provide a set of $p$ (from 2 to $n-1$ ) modules partitions.

3. In each B bootstrap sample and $p$ value, a ${ }^{\mathrm{B}} \boldsymbol{r}_{\boldsymbol{p}}$ vector of $\left(n^{2}-n\right) / 2$ relevant, non redundant variable cluster allocation coincidences is obtained. Then the variance of $\boldsymbol{r}_{\boldsymbol{p}}$ across bootstrap samples and the sum of variances in partition $p$ are calculated.

4. The previous process is repeated for randomized, no-correlation samples of the original data set, to generate a distribution of the sum of variances for $p$-module partitions in the absence of any module structure. These averages of these distributions are used to normalize the sums obtained for the same $p$ value in 5), and its percentiles to test for the existence of module structure for that partition.

5. The partition showing the lowest normalized sum of variances -variance criterion- is taken as the best clustering solution.

\section{Module identification}

The performance of BoCluSt was studied in simulated datasets of grouped variables $x_{i j}$ :

$$
x_{i j}=c_{i}+e_{i j}
$$

where variable component $c_{i}$ was common to all $x$ variables in module $I$, causing correlation among these variables, and $e_{i j}$ was specific to each $x_{i j}$. The considered datasets are shown in Table 1.

\section{Detection of hierarchical modular structures}

The ability of BoCluSt to detect hierarchical modular structures (i. e., the presence of sub-modules within modules) was studied by simulating datasets of variables:

$$
x_{i j k}=g_{i}+s_{i j}+e_{i j k}
$$

where $\mathrm{g}_{\mathrm{i}}, \mathrm{s}_{\mathrm{ij}}$ and $\mathrm{e}_{\mathrm{ijk}}$ were module, sub-module and variable-specific, respectively.

\section{Comparison with other procedures: computer simulation}

Multi-sample simulations compared BoCluSt with the algorithm by Ahn et al [37] based on clustering the links between nodes instead of the nodes themselves and aimed at the detection of hierarchical and overlapping community structures, as implemented in the R CRAN package linkcomm [38]; with the standard silhouette cluster validation procedure [39], based on ratios of between-node distances, as implemented in the cluster R CRAN package; and with the community detection procedures in the igraph R CRAN package [40]. These were the Edge betweenness [41], a divisive method based on modularity; Fastgreedy [42], a hierarchical 
Table 1. Cases considered in the computer simulations in Fig 1.

\begin{tabular}{|c|c|c|c|c|c|c|}
\hline Case & Number of variables & Number of modules & Module sizes & Sample size & Components distribution & $v(c)$ \\
\hline a & 8 & 2 & 4,4 & 100 & Normal & 0.030 \\
\hline b & 8 & 4 & $2,2,2,2$ & 100 & Normal & 0.030 \\
\hline c & 8 & 2 & 4,4 & 100 & Normal & 0.010 \\
\hline$d$ & 8 & 2 & 4,4 & 100 & Normal & 0.015 \\
\hline e & 8 & 2 & 4,4 & 25 & Normal & 0.030 \\
\hline f & 8 & 2 & 4,4 & 50 & Normal & 0.030 \\
\hline g & 8 & 7 & $1,1,1,1,1,1,2$ & 100 & Normal & 0.030 \\
\hline $\mathrm{h}$ & 8 & 3 & $5,2,1$ & 100 & Normal & 0.030 \\
\hline i & 8 & 2 & 4,4 & 100 & Beta & $0.030^{*}$ \\
\hline j & 8 & 2 & 4,4 & 100 & Uniform & $0.030 * *$ \\
\hline k & 4 & 2 & 2,2 & 100 & Normal & 0.030 \\
\hline I & 16 & 2 & 8,8 & 100 & Normal & 0.030 \\
\hline
\end{tabular}

The cases differed in number of variables, number and sizes of modules (there were as many modules as sizes listed), number of individuals measured, distributions of the variables and variance of $c, v(c)$. In all cases, e had a variance of 0.050 . The correlations corresponding to the three considered $v(c)$ values were $0.375,0.231$ and 0.167 .

${ }^{*} c$ was generated as a beta variable with parameters $\alpha=0.246$ and $\beta=2$, and $e$ as a beta variable with $\alpha=0.625$ and $\beta=2$, using $R$ function $r b e t a$; the resulting $x, c$ and $e$ distributions were markedly asymmetric.

** $c$ was generated as a uniform variable with the range 0 to 0.600 and $e$ as a uniform variable with the range 0 to 0.775 using $R$ function runif.

doi:10.1371/journal.pone.0156576.t001

agglomeration algorithm based on modularity; Infomap, which finds the community structure that minimizes the expected description length of a random walker trajectory [43]; Label propagation [44], a nearly linear time algorithm that labels the network nodes and then updates these labels by majority voting in the neighborhood of the node; Leading eigenvector [45], based on calculating the leading non-negative eigenvector of the modularity matrix of a graph; the "Louvain procedure" [46], which finds community structure by optimizing modularity; Optimal modularity [47], which tries to maximize the modularity measure over all possible community partitions; Spinglass [48], based on simulated annealing, and Walktrap [49], which uses random walks on graphs to detect densely connected subgraphs, i. e., communities. These compared procedures took lists of weighted links between nodes-variables as input; here, the weights were the between-variables Pearson correlations calculated with the data generated in each simulation iteration.

\section{Comparison with other procedures: Analysis of gene expression data}

These community detection procedures were also applied to the transcriptomic data in [50], corresponding to measures of nuclear gene expression in a Drosophila melanogaster experiment in which five different mitochondrial strains were introgressed in flies with an identical nuclear genetic background (NCBI accession GSE24729; CEL files, 40 samples; available at: http://www.ncbi.nlm.nih.gov/geo/query/acc.cgi?acc=GSE24729). Using AmiGO 2 [51] and Bioconductor affy ([52]; RMA normalization method), annaffy [53], and drosophila2.db [54] libraries, the expression of two sets of genes were analyzed. First, of all, genes involved in cell growth proliferation, annotated with the ontologies GO:0030307, "positive regulation of cell growth" and GO:0030308, "negative regulation of cell growth". It was possible to obtain expression measures for 24 probe sets corresponding to 22 genes in these ontologies. Secondly, the larger set of genes annotated as "DNA repair" (GO:0006281; 104 probesets in the data file, corresponding to 100 genes). 


\section{Results and Discussion}

BoCluSt was able to identify the correct number of modules even for small-sized samples and also moderate correlations between variables in the same module (Fig 1). Thus, a sample size of 25 (Fig 1e) was enough to easily identify two modules of four variables with a within-module correlation of 0.375 , and modules with within-module correlation of 0.231 were easily detected using samples with a size of 100 (Fig 1d). The performance of the procedure did not obviously depend on module number and size, the homogeneity of these sizes (Fig $1 \mathrm{~g}$ and $1 \mathrm{~h}$ ) or the variables' distributions (Fig $1 \mathrm{i}$ and $1 \mathrm{j}$ ). The less favorable situations were those with the lowest correlation within modules (0.167, Fig $1 \mathrm{c}$ ) and the lowest number of variables (four variables, Fig $1 \mathrm{k})$. In the latter case, the variance criterion was clearly under the corresponding value for the null case, but the differences between the two and the three clusters solutions were very slight.

Fig 1 considers only from 2 to $n$ - 1 as the possible cluster numbers because it makes little sense to consider any coincidences in module allocation when there are $n$ clusters of size one (and therefore no coincidences) or when there is a single cluster including all variables (total coincidence). However, because BoCluSt compares the obtained results with those expected under the absence of community structure, it is possible to conclude that there is no evidence for such structure when all the 2 to $n$ - 1 partitions are above the lower 2.5 percentile of the null distribution. Thus, BoCluSt does not only provide an estimate of the number of modules, but also of the reliability of that estimate and of the overall degree of modular structure in the data. It also makes it possible to compare the amount of evidence for alternative solutions.

BoCluSt was able to detect hierarchical modular structures, especially when the hierarchy was regular, i. e., the pattern of subdivision was the same in all modules (Fig 2a, 2d and 2e). These regular partitions appeared as local minima in the variance criterion profile: two and four modules in Fig 2a; two, four and eight modules in Fig $2 \mathrm{~d}$. The procedure failed in the case of four modules and eight sub-modules (Fig 2e), in which the second local minimum was found for nine clusters instead of eight. This suggests that the sample size required for module identification increases with module number. In any case, it must be noted that, while able to detect some hierarchical structures, BoCluSt does not provide a formal diagnostic for such structures.

Defining a single correct result became harder for less regular partitions. For example, in Fig $2 \mathrm{~b}$, both two or three module partitions would be possible. While the partition into two modules was easily detected, that into three modules resulted in a local maximum instead of a minimum. This maximum disappeared when the correlation between variables in the large module on the right of the diagram increased (Fig 2c), which, not unexpectedly, suggests that module detection is easier when within module links are strong. In any case, the low criterion values for two and three clusters seen in Fig 2c are not be clear evidence of hierarchical clustering, because the criterion values neighboring a minimum can also be low in non-hierarchical situations, as seen for example in Fig $1 \mathrm{~h}$ and $1 \mathrm{k}$, or in the case of partially overlapping modules.

Fig 2e shows many consecutive low values for the variance criterion. This could be related to the fact that many partitions (into four, five, six or eight modules) are possible in that case. However, this could not explain all results. The criterion values remained low beyond eight, the last "correct" number of clusters. In any case, the comparison of Figs 1 and 2 suggests that profiles showing more than one point of inflexion may be indicators of hierarchical modular structures.

In the computer simulation comparisons between procedures, the BoCluSt, silhouette, Louvain and Walktrap procedures never failed to identify two modules for sample sizes of one hundred and moderate within module correlations of 0.375 (Fig 3 2C3). Louvain and Walktrap were better than BoCluSt when the correlation was reduced to 0.167 (Fig 3 2C1). However, all 


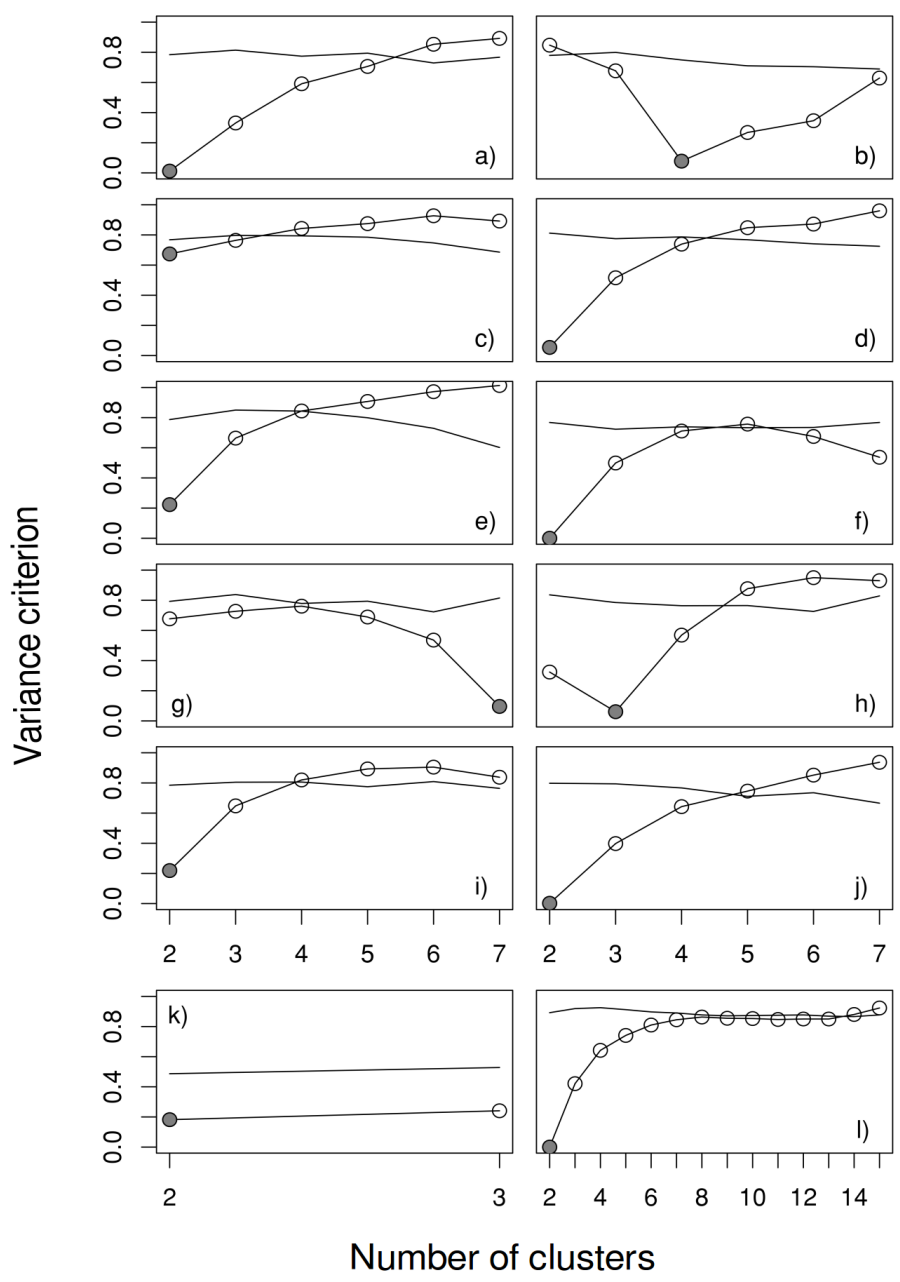

Fig 1. Values for the variance criterion (circles) in the cases listed in Table 1. A single, randomly taken simulated data set is shown per case, with 100 randomized null data sets and 500 bootstrap resamples per data set, along with the lower 2.5 percentile (simple lines) for the corresponding null situation of no correlation between variables. Grey circles mark the value for the true number of modules.

doi:10.1371/journal.pone.0156576.g001

procedures except silhouette were clearly worse than BoCluSt in the case of four modules, as they failed to find four modules as the most frequent result when the correlation was 0.375 (Fig $34 \mathrm{C} 3$ ) and completely failed to detect them when the correlation was 0.167 (Fig $34 \mathrm{Cl}$ ). In the same situations, the right solution of four modules was that most frequently found by BoCluSt. The silhouette procedure slightly surpassed BoClust in performance in the 4C 3 case but tended to underestimate the number of clusters when the correlations within clusters decreased in the $4 \mathrm{C} 1$ case.

In the hierarchical cases, most procedures tended to find only one or two modules in most replicates. The silhouette procedure quite frequently obtained an intermediate wrong result of three modules, whereas BoCluSt found two and four modules as the most frequent solutions. It was able to detect the hierarchical structure in most replicates when the correlation was moderate (Fig $32 / 2 \mathrm{C} 3$, in italics), but only in a minority when the correlation was low (Fig 3 2/2C1). The Edge betweenness and Label propagation procedures did particularly badly in the small number of variables cases considered in these simulations. This was also the case for Infomap and linkcomm, which failed to find any module structure in almost all simulation 


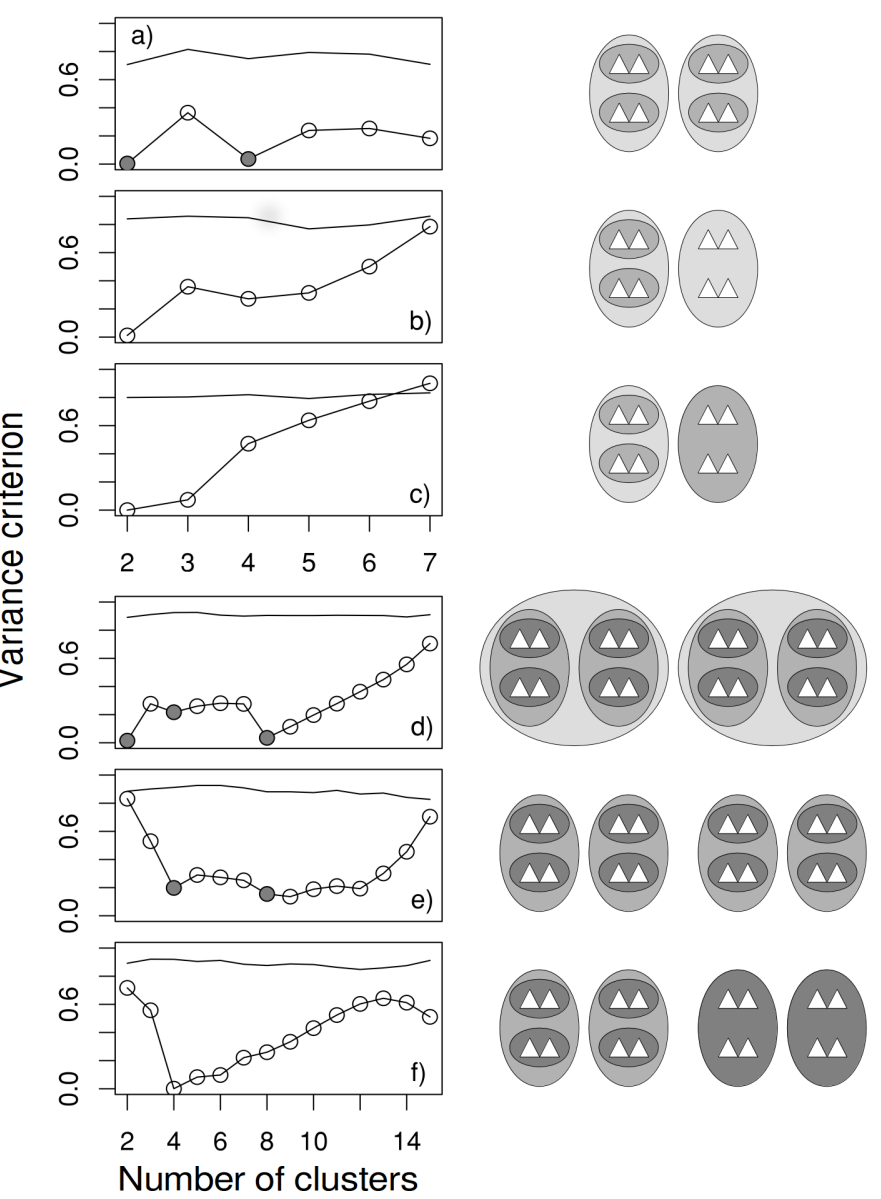

Fig 2. Analysis of hierarchical communities. A single, randomly taken simulated data set is shown per case, with 100 records, 100 randomized null data sets and 500 bootstrap resamples per data set. The graphs (left) show the variance criterion for all possible numbers of clusters along with the lower 2.5 percentile for the corresponding null situation of no correlation between variables (simple lines). Grey circles mark correct clustering results for regular partitions. The diagrams to the right represent the different situations. The grayscale indicates the value of the correlation between variables (triangles) in the same ellipse. These were 0.273 and 0.545 in the eight variable cases $(a-c)$, and $0.214,0.429$ and 0.643 in the 16 variable cases (d to f).

doi:10.1371/journal.pone.0156576.g002

replicates. This was due to the maximum link density in these data sets. The removal of lowweight (i. e., low correlation) links improved markedly both procedures' ability to detect modules (not shown). However, in a practical situation this filtering would require to set an arbitrary threshold for link weights, which would limit the procedures' utility for unsupervised module detection.

BoCluSt outperformed the other procedures in most simulations made here, especially in small module cases. The low performance of some of these procedures was likely related to the fact that they are based on measuring modularity. Modularity-based methods face a "resolution limit in community detection", which is most likely to occur when the number of module internal links is of the order of the square root of twice the total number of links in the network or smaller [55], i. e., when modules are small. BoCluSt seems to be unaffected by that limit, since it can easily detect two-variables modules (see Fig 1b). However, the use of bootstrap resampling by this procedure might command too many computational resources to be practical for the analysis of data sets of many variables, as those in genome-wide or human social networks. Thus, BoCluSt could be an alternative to low computational complexity, large-scale procedures 


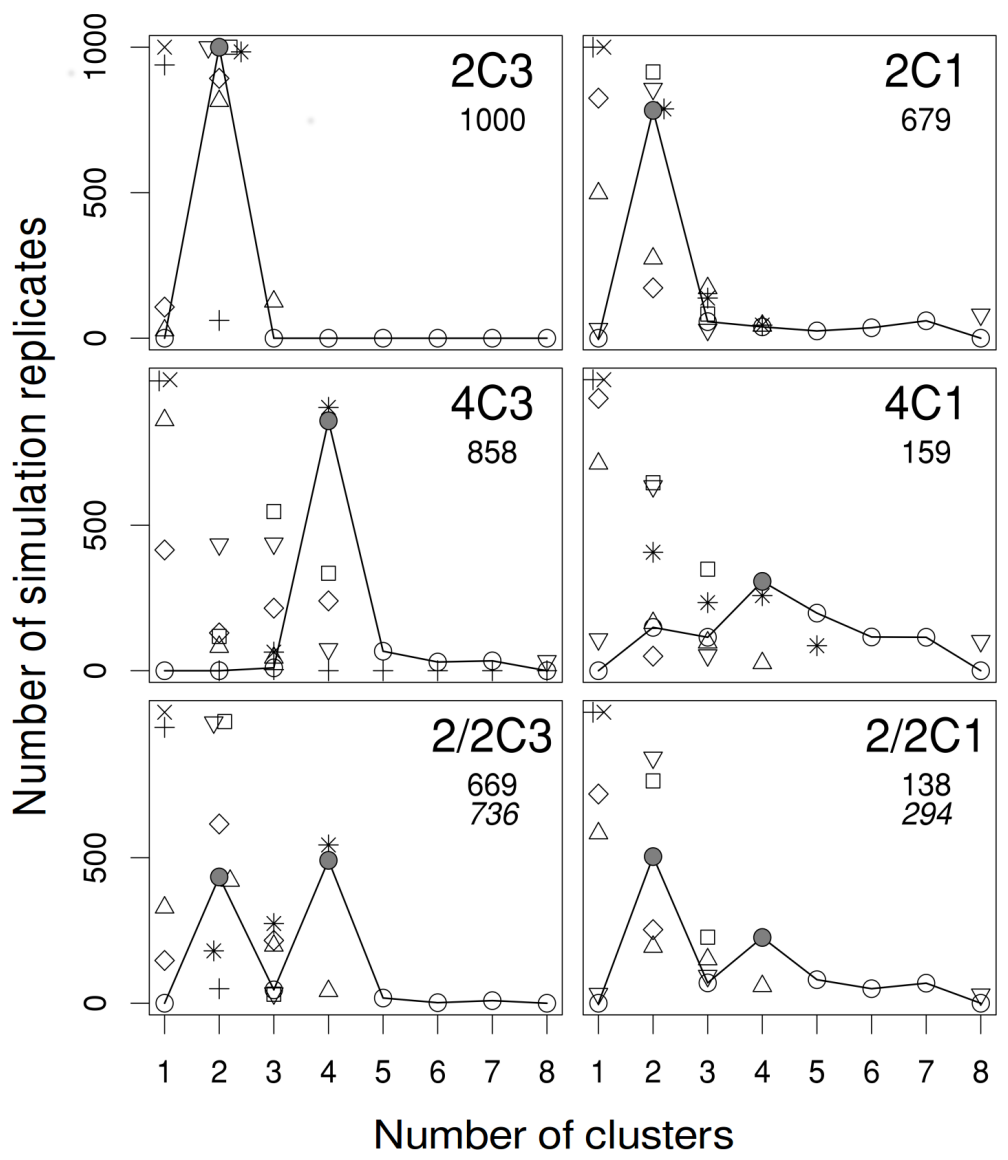

Fig 3. Frequency of numbers of modules detected in computer simulations comparing the different procedures. There were eight variables, 100 records and 1000 replicates per case. $2 \mathrm{C}$ and $4 \mathrm{C}$ are cases with two and four variable modules respectively, and $2 / 2 \mathrm{C}$, hierarchical situations of two modules each divided in two sub-modules. The numbers to the right of the Cs mark the variance of component $c$, common to variables in the same module or sub-module: 1 , variance $=0.01 ; 3$, variance $=0.03$ (the variance of component $e$ was $=0.05$ in all cases). Circles, upwards triangles, plus signs, $x$ signs, rhombs, squares, asterisks, and downwards triangles show results for the BoCluSt, Edge betweeness, Infomap, Label propagation, linkcomm, Louvain, silhouette, and Walktrap procedures, respectively. In these simulations, the results for Fastgreedy, Leading eigenvector, Optimal modularity and Spinglass procedures were very similar to those of Louvain; only the latter is shown for clarity. Also for clarity, counts lower than 25 are shown for BoCluSt only. Grey symbols mark the true number(s) of modules. The smaller font values are the numbers of replicates in which BoCluSt found results both correct and significant (under the 2.5 quantile of the null distribution). In the hierarchical cases, these results consisted in two significant minima in the variance criterion for two and four clusters. In italics, the number of replicates finding the same two minima, whether significant or not.

doi:10.1371/journal.pone.0156576.g003

in the analysis of moderate-sized data sets. The compared procedures would not be equivalent to BoCluSt for any problem size, however, because they do not use the same kind of information. Instead of starting with a previously known set of link weights, BoCluSt simultaneously estimates both weights and community structure.

In the analysis of the expression of genes involved in cell growth regulation (Fig 4 up; kmeans analysis; 100 bootstrap replicates for both the observed and the randomized null cases; 100 such null cases were done), BoCluSt found clear evidence for three modules (the variance criterion for this solution was zero, showing that variables were placed in the same three clusters in all bootstrap resamples), and also for a hierarchical modular structure, as the results suggested that the three modules were composed of 15 and 23 sub-modules. 


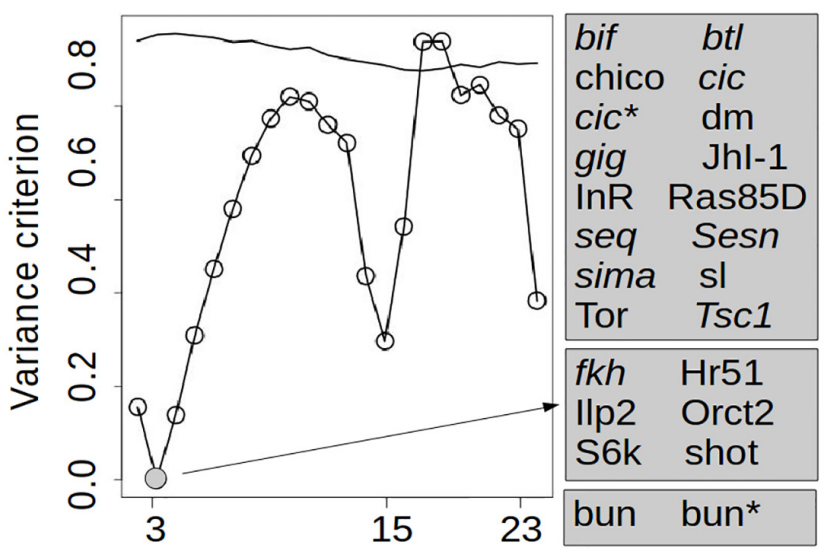

Number of clusters
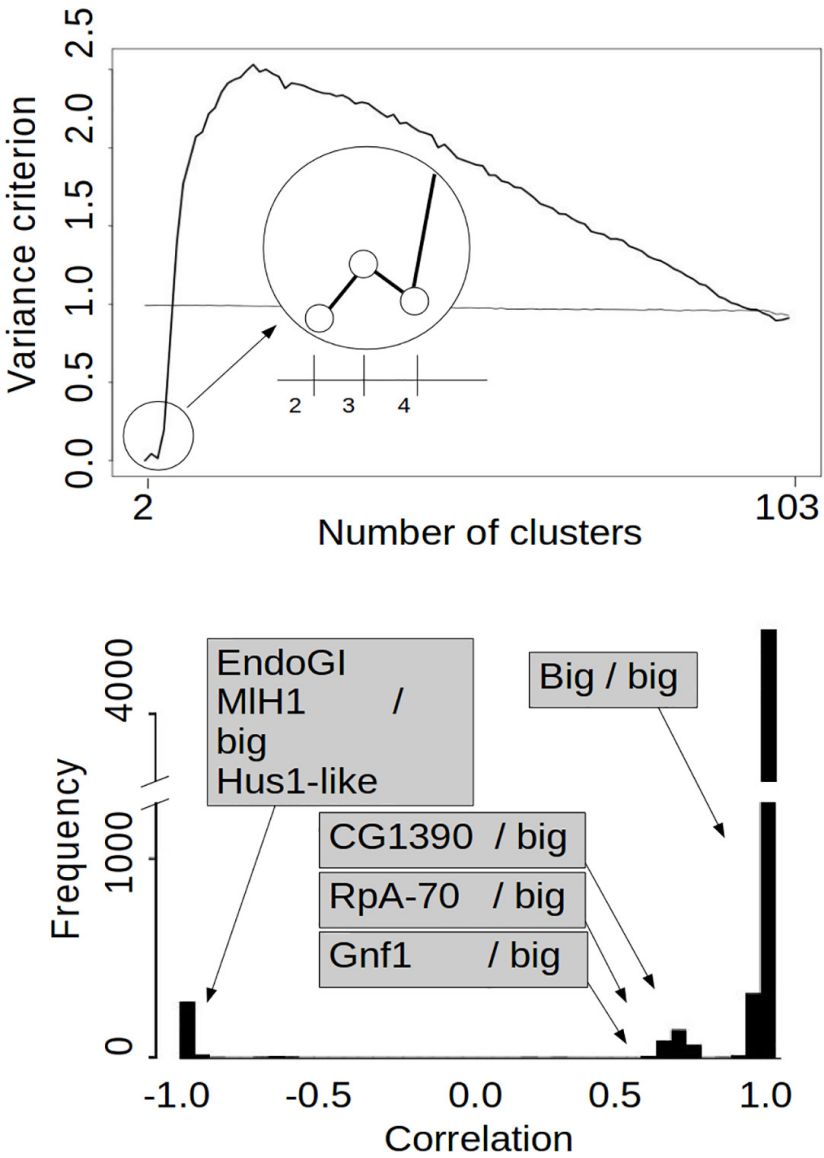

Fig 4. BoCluSt analysis of Drosophila melanogaster microarray data. Top, analysis of probes for 23 genes annotated in the ontologies "positive control of cell growth" and "negative control of cell growth" (in normal and italic fonts respetively). The gene composition of the three-clusters partition with the least value for the variance criterion is shown in the grey boxes to the right of the graph. Asterisks mark second probes of genes having two different probes in the array. Middle, analysis of 104 probe sets of genes annotated in the ontology "DNA repair". The partition showing the lowest variance criterion is amplified in the circle. Bottom, frequencies of pairwise correlation values between the probe sets corresponding to DNA repair genes (5356 correlations among 104 probe sets). The analysis identified a big cluster of 98 genes of narrowly correlated expressions. The bars correspond to the correlations a) of these 98 probe sets among themselves ("Big/big" box); b) of these probe sets with the EndoGI, MIh1 and Hus1-like probe sets (left box); and c) with the CG1390, RpA-70 and Gnf1 probe sets (the three boxes at center; see the text for more detailed explanations).

doi:10.1371/journal.pone.0156576.g004 
Most compared procedures tended to detect only two groups, i. e., they did not detect a third cluster including the two probe sets corresponding to gene bun (Table 2). Only Walktrap found the same structure as BoCluSt.

In the case of the DNA repair genes, BoCluSt found a global minimum variance criterion for two clusters, and a local minimum for four clusters (Fig 4, middle). The correlation structure for the expressions of these probes was remarkably simple (Fig 4, bottom), which makes it easier to discuss the clustering results. There were 98 narrowly and positively correlated ( $\mathrm{r}>0.8)$ probe sets, three probe sets (corresponding to genes CG10694, cry and qjt) tightly correlated $(r>0.96)$ among themselves and with strong negative correlations $(r<-0.88)$ with the previous 98, and finally three probe sets corresponding to genes Blm, CG18004 and $p 53$ with positive correlations (from 0.55 to 0.73 ) with the big group of 98 , negative correlations with the previous set of three (from -0.73 to -0.62 ), and moderate ones among themselves ( 0.19 to 0.61 ). Notice that the visible bars in the histogram in Fig 4 correspond to the numerous correlations involving the big group of 98 probe sets; the correlations among the other 6 probe sets were too few for visible bars. Thus, three "bar islands" can be seen in the histogram: on the left, the correlations of CG10694, cry and qjt with the variables in the big group, in the center, the correlations of Blm, CG18004 and $p 53$ with the big group, and on the right, the correlations within the big group.

The BoCluSt results describe this structure. The solution with the largest support (two clusters) separates cry, CG10694 and qjt, narrowly correlated among themselves and negatively correlated with the rest. In the also well supported solution of four clusters, CG18004 and p53 are separated from the larger group to constitute two single-variable clusters, which is not unexpected given that the two genes are not narrowly correlated to each other. Blm separates last, again in a single variable (fifth) cluster. It is not obvious, however, why the variance criterion increased markedly for this five-clusters solution (Fig 4, middle), given that the correlations for $B l m$ were similar to those of the previous two.

In any case, BoCluSt provided a clearly better description of the community structure among these 104 probe sets than the compared procedures (Table 2), which, for example, included genes cry, CG10694 and qjt and the negatively correlated Blm in the same cluster (Louvain procedure); allocated these three first genes in different clusters, despite the extreme positive correlations among them (Edge betweenness); or included Arp5, which had a correlation profile typical of genes in the big group of correlated genes, in a second cluster of seven genes (Leading eigenvector). Only Walktrap found the same solution as BoCluSt, as it had done for the smaller analysis of Cell Growth genes. However, BoCluSt and Walktrap are not equivalent. First, as shown by the computer simulations, Walktrap tended to fail as the number of communities increased (Fig 3); second, it does not take into account the precision in the calculation of link weights, and third, it could not detect hierarchical communities (igraph provides the modularity measures corresponding to all cluster partitionings of the variables -not shown, and no clear local minima is seen in this series of measurementss for any of the microarray data sets, in contrast with what can be seen in Fig 4 for BoCluSt). The silhouette procedure did not reflect the correlation structure discussed above. The RpLPO and Tctp genes constituting the second cluster identified by this procedure had expressions which were very similar (correlations 0.97) to those of genes allocated to the Big group by the remaining procedures, and high and negative correlations $(<-0.93)$ with genes CG10694, cry and qjt. These poor results were to some extent unexpected, as silhouette works best in situations with compact, clearly separated and roughly spherical clusters [39], as was apparently the case with these DNA repair genes data. One possibility is that the performance of silhouette was impaired by the very large differences in module size. As already observed for cell growth regulation genes, Infomap and linkcomm (and also Label propagation) were unable to detect any module structure. This could 
Table 2. Number and sizes of clusters found by the compared procedures in the analysis of gene expression.

\begin{tabular}{|c|c|c|c|c|}
\hline \multirow[t]{2}{*}{ Procedure } & \multicolumn{2}{|c|}{ Cell growth } & \multicolumn{2}{|c|}{ DNA repair } \\
\hline & \# Clusters & Sizes & \# Clusters & Sizes/Composition \\
\hline BoCluSt & 3 & $16,6,2$ & 2 & (Big), (c, $\left.\mathrm{C}_{1}, \mathrm{q}\right)$ \\
\hline Edge betweeness & 8 & $17,1,1,1,1,1,1,1$ & 4 & $(\mathrm{Big}),(\mathrm{c}),\left(\mathrm{C}_{1}\right),(\mathrm{q})$ \\
\hline Fastgreedy & 2 & 16,8 & 1 & - \\
\hline Infomap & 1 & 24 & 1 & - \\
\hline Label propagation & 1 & 24 & 1 & - \\
\hline Leading eigenvector & 2 & 16,8 & 2 & (Big), (A, B, c, $\left.C_{1}, C_{2}, p, q\right)$ \\
\hline Linkcomm & 1 & 24 & 1 & - \\
\hline Louvain & 2 & 16,8 & 2 & $(B i g),\left(A, c, C_{1}, q\right)$ \\
\hline Optimal modularity & 2 & 16,8 & 2 & (Big), $\left(B, c, C_{1}, q\right)$ \\
\hline silhouette & 2 & 15,9 & 2 & (Big), (T, R) \\
\hline Spinglass & 2 & 16,8 & 2 & (Big), (B, c, $\left.C_{1}, q\right)$ \\
\hline Walktrap & 3 & $16,6,2$ & 2 & (Big), (c, $\left.\mathrm{C}_{1}, \mathrm{q}\right)$ \\
\hline
\end{tabular}

The simpler partitions found for genes in the "DNA repair" ontology made it possible to list the genes in the smaller clusters (A, Arp5; B, BIm; $\mathrm{C}_{1}$, CG10694; $\mathrm{C}_{2}$, CG18004; c, cry; p, p53; q, qjt; R, RpLP0; T, Tctp; Big, all genes in the data set but those listed for each procedure). Genes within the same parenthesis set were in the same cluster.

doi:10.1371/journal.pone.0156576.t002

be related to link density, which was rather extreme in this data set, including 98 narrowly correlated variables. As mentioned above, the performance of these two procedures in the computer simulations was much better when link density was artificially reduced. This highlights the fact that the comparisons made in this article had to use the particular data structure required by BoCluSt, which could partly explain the advantage found for this procedure.

BoCluSt uses a file of individual observations for some variables as input and then estimates the weights of all possible links between these variables. Thus, it is designed to work at maximum link density, whereas the alternative procedures were designed to use a limited list of links and weights from somewhat sparse networks. Therefore, it is possible that these procedures do not work at their best when applied to the full density link sets specified by correlation matrices, as done here. However, this full-density, exploratory community detection considering all possible links between variables may be appropriate in a wide range of situations, such as the gene expression studies considered here.

\section{Conclusions}

BoCluSt may be a valuable and robust tool in community detection analysis, as it provides 1) an unsupervised estimation of the number of modules and their composition; 2) a measure of the amount of evidence for this estimation and alternative module partitions, and 3) an overall description of the modular structure of the whole data set, which may reveal the existence of hierarchies of modules and nested sub-modules. Because of its unsupervised nature, BoCluSt can be used in automated analyses or in comparisons between data sets. The procedure uses kmeans or pam as clustering algorithms in its present implementation, but its method of evaluating alternative partitions based on measuring their stability under resampling, in principle, would be compatible with any clustering algorithm. While permitting a more detailed analysis of community structure and the evaluation of alternative module partitions, its increased computational complexity would restrict its use to small to moderate data sets. However, this article shows that it tends to outperform alternative procedures for these kinds of data sets 
when network density is high. BoCluSt code is written in $\mathrm{R}$ and available at http://sourceforge. net/projects/boclust/files/BoCluSt.txt/download It requires the use of the pam function from cluster R package in case the Partition About Medoids clustering algorithm is chosen.

\section{Acknowledgments}

I would like to thank M. Elena Vazquez-Abal for help with the procedure description.

\section{Author Contributions}

Conceived and designed the experiments: CG. Performed the experiments: CG. Analyzed the data: CG. Contributed reagents/materials/analysis tools: CG. Wrote the paper: CG.

\section{References}

1. Proulx SR, Promislow DE, Phillips PC. Network thinking in ecology and evolution. Trends Ecol Evol. 2005; 20:345-53. PMID: 16701391

2. Boccaletti S, Latora V, Moreno Y, Chavez M, Hwang D. Complex networks: Structure and dynamics. Phys Rep. 2006; 424: 75-308.

3. von Dassow G, Munro E. Modularity in animal development and evolution: elements of a conceptual framework for Evo-Devo. J Exp Zool B Mol Dev Evol. 1999; 285:307-25.

4. Callebaut W, Rasskin-Gutman D. Modularity: Understanding the development and evolution of natural complex systems. Cambridge: MIT Press; 2005.

5. Wagner GP, Pavlicev M, Cheverud JM. The road to modularity. Nat Rev Genet. 2007; 8:921-31. PMID: 18007649

6. Hintze A, Adami C. Evolution of complex modular biological networks. PLoS Comput Biol. 2008; 4:e23 doi: 10.1371/journal.pcbi.0040023 PMID: 18266463

7. Segal E, Friedman N, Koller D, Regev A. A module map showing conditional activity of expression modules in cancer. Nat Genet. 2004; 36:1090-8. PMID: 15448693

8. Litvin $\mathrm{O}$, Causton $\mathrm{HC}$, Chen $\mathrm{BJ}$, Pe'er $\mathrm{D}$. Modularity and interactions in the genetics of gene expression. P Natl Acad Sci USA. 2009; 106: 6441-6.

9. Spirin V, Mirny LA. Protein complexes and functional modules in molecular networks. P Natl Acad Sci USA. 2003; 100:12123-8.

10. Ravasz E., Somera AL, Mongru DA, Oltvai ZN, Barabási A. Hierarchical organization of modularity in metabolic networks. Science. 2002; 297:1551-5. PMID: 12202830

11. Kreimer A, Borenstein E, Gophna U, Ruppin E. The evolution of modularity in bacterial metabolic networks. P Natl Acad Sci USA. 2008; 105:6976-81.

12. Wagner GP. Homologues, natural kinds, and the evolution of modularity. Am. Zool. 1996; 36:36-43.

13. Bolker JA. Modularity in development and why It matters to Evo-Devo. Amer. Zool. 2000; 40:770-6.

14. Jordano $P$. Patterns of mutualistic interactions in pollination and seed dispersal: connectance, dependence asymmetries, and coevolution. Am Nat. 1987; 129:675-7.

15. Dupont YL, Olesen JM. Ecological modules and roles of species in heathland plant-insect flower visitor networks. J Anim Ecol. 2009; 78:346-53. doi: 10.1111/j.1365-2656.2008.01501.x PMID: 19021779

16. Fortunato $S$. Community detection in graphs. 2010; Physics Reports 486:75-174.

17. Klingenberg CP. Morphometric integration and modularity in configurations of landmarks: tools for evaluating a priori hypotheses. Evol Dev. 2009; 11:405-21. doi: 10.1111/j.1525-142X.2009.00347.x PMID: 19601974

18. Fruciano $C$, Franchini $P$, Meyer A. Resampling-based approaches to study variation in morphological modularity. PLoS ONE. 2013; 8:e69376. doi: 10.1371/journal.pone.0069376 PMID: 23874956

19. Guimera R, Nunes Amaral LA. Functional cartography of complex metabolic networks. Nature. 2005; 433:895-900. PMID: 15729348

20. Duch J, Arenas A. Community detection in complex networks using extremal optimization. Phys Rev E. 2005; 72:027104.

21. Sales-Pardo M, Guimerã R, Moreira AA, Amaral LAN. Extracting the hierarchical organization of complex systems. P Natl Acad Sci USA. 2007; 104:15224-9. 
22. Barrat $A$, Barthélemy $M$, Pastor-Satorras $R$, Vespignani $A$. The architecture of complex weighted networks. P Natl Acad Sci USA. 2004; 101:3747-52.

23. Newman MEJ. Modularity and community structure in networks. P Natl Acad Sci USA. 2006; 103:8577-82.

24. Girvan M, Newman MEJ. Community structure in social and biological networks. P Natl Acad Sci USA. 2002; 99:7821-6.

25. Zhou H. Network landscape from a brownian particle's perspective. Phys RevE. 2003; 67: 041908.

26. Handcock MS, Raftery AE, Tantrum JM. Model based clustering for social networks. J. Roy. Statist. Soc. A. 2007; 170:1-22. Working Paper no. 46.

27. Breckenridge J. Replicating cluster analysis: method, consistency and validity. Multivar. Behav. Res. 1989; 24:147-161.

28. Falasconi M, Gutierrez A, Pardo M, Sberveglieri G, Marco S. A stability based validity method for fuzzy clustering. Pattern Recogn. 2010; 43:1292-305.

29. Levine E, Domany E. Resampling method for unsupervised estimation of cluster validity. Neural Comput. 2001; 13:2573-93. PMID: 11674852

30. Lange T, Roth V, Braun ML, Buhmann JM. Stability-based validation of clustering solutions. Neural Comput. 2004; 16:1299-323. PMID: 15130251

31. Borgelt C. Resampling for fuzzy clustering. Int J Uncertain Fuzz. 2007; 15:595-614.

32. Law M, Jain A. Cluster validity by bootstrapping partitions. Technical Report MSU-CSE-03-5. Department of Computer Science and Engineering, Michigan State University, 2002.

33. Jain AK, Dubes RC. Algorithms for clustering data. Prentice Hall Englewood Cliffs, N. J; 1988.

34. R Development Core Team. R: A language and environment for statistical computing. R Foundation for Statistical Computing, Vienna, Austria. ISBN 3-900051-07-0. Available: http://www.R-project.org.; 2008

35. Jain AK. Data clustering: 50 years beyond K-means. Pattern Recog Lett. 2010; 31:651-666.

36. Maechler M, Rousseeuw P, Struyf A, Hubert M, Hornik K. cluster: Cluster Analysis Basics and Extensions. R package version 2.0.1. 2015. Available: http://cran.r-project.org.

37. Ahn Y, Bagrow JP, Lehmann S. Link communities reveal multiscale complexity in networks. Nature. 2010; 466:761-764. doi: 10.1038/nature09182 PMID: 20562860

38. Kalinka AT, Tomancak P. linkcomm: an R package for the generation, visualization, and analysis of link communities in networks of arbitrary size and type. Bioinformatics. 2011;27:2011-2012. doi: 10.1093/ bioinformatics/btr311 PMID: 21596792

39. Rousseeuw PJ 1987. Silhouettes: A graphical aid to the interpretation and validation of cluster analysis. J Comput Appl Math. 1987; 20:53-65.

40. Csárdi G, Nepusz T. The igraph software package for complex network research. InterJournal, Complex Systems. 2006;1695. Available: http://igraph.org

41. Newman M, Girvan M. Finding and evaluating community structure in networks. Phys Rev E. 2004; 69:026113.

42. Clauset A, Newman MEJ, Moore C. Finding community structure in very large networks. Available: http://www.arxiv.org/abs/cond-mat]

43. Rosvall $\mathrm{M}$, Bergstrom $\mathrm{CT}$. Maps of information flow reveal community structure in complex networks. Proc Natl Acad Sci USA. 2008; 105;1118-1123.

44. Raghavan UN, Albert R, Kumara S. Near linear time algorithm to detect community structures in largescale networks. Phys Rev E. 2007; 76:036106.

45. Newman MEJ. Finding community structure using the eigenvectors of matrices, Phys Rev E. 2006; 74:036104.

46. Blondel VD, Guillaume JL, Lambiotte R, Lefebvre E. Fast unfolding of communities in large networks. J Stat Mech Theory Exp. 2008; doi: 10.1088/1742-5468/2008/10/P10008

47. Brandes U, Delling D, Gaertler M, Gorke R, Hoefer M, Nikoloski Z, Wagner D. On Modularity Clustering. IEEE Transactions on Knowledge and Data Engineering. 2008; 20:172-188.

48. Reichardt J, Bornholdt S. Statistical Mechanics of Community Detection. Phys. Rev. E. 2006; 74:016110.

49. Pons $\mathrm{P}$, Latapy M. Computing communities in large networks using random walks. Available: http:// arxiv.org/abs/physics/0512106] 
50. Innocenti P, Morrow EH, Dowling DK. Experimental evidence supports a sex-specific selective sieve in mitochondrial genome evolution. Science. 2011; 332:845-848. doi: 10.1126/science.1201157 PMID: 21566193

51. Carbon S, Ireland A, Mungall CJ, Shu S, Marshall B, Lewis S, AmiGO Hub, Web Presence Working Group. AmiGO: online access to ontology and annotation data. Bioinformatics. 2009; 25:288-289. doi: 10.1093/bioinformatics/btn615 PMID: 19033274

52. Gautier L, Cope L, Bolstad BM and Irizarry RA. affy-analysis of Affymetrix GeneChip data at the probe level. Bioinformatics. 2004; 20:307-315. PMID: 14960456

53. Smith CA. annaffy: Annotation tools for Affymetrix biological metadata. R package version 1.38.0. 2010.

54. Carlson M. drosophila2.db: Affymetrix Drosophila Genome 2.0 Array annotation data (chip drosophila2). $R$ package version 3.1.2.

55. Fortunato S, Barthelemy M. Resolution limit in community detection. P Natl Acad Sci USA. 2007; 104:36-41. 